\title{
Complex Dynamic States in Multi-phase Traffic Model
}

\author{
R. Nagai and T. Nagatani \\ Department of Mechanical Engineering, Shizuoka University, 3-5-1 Johoku, Hamamatsu, 432-8561 Japan
}

A multi-phase traffic model is presented to take into account the complex motion of vehicles. The jamming transition of traffic flow is very similar to the conventional phase transitions and critical phenomena[1]. In the fundamental model, there are two traffic phases and their coexisting phase : free flow, homogeneous congested flow, and inhomogeneous jammed flow. They correspond, respectively, to gas, liquid, and coexisting phase in the gas-liquid phase transition. However, real traffic exhibits a very rich variety of phenomena. The fundamental model result is in a conflict with empirical evidence[2]. The multi-phase model is an extended one of the optimal velocity model. The optimal velocity (OV) function is modified to have multi phases.

Fig.1 shows the optimal velocity functions with a single stage and two stages. The original OV model with a single stage exhibits two phase traffic, while the model with $n$ stages displays $n+1$ phase traffic.

The phase transitions depend highly on the sensitivity (the inverse of delay time). The neutral stability line is obtained by using the linear stability analysis and is consistent with the jamming transition points. The phase diagrams are presented for the multiple jamming transitions. Fig.2 shows the phase diagram for the OV model with two stages. Phase diagram in the phase space for three-phase model is presented. The circles and squares indicate the phase separation lines. The dotted line indicates the spinodal line. Phases 1, 2, and 3 appear, respectively, in regions V, III, I. The coexisting phase of phases 1 and 2 occurs in region IV. The coexisting phase of phases 2 and 3 occurs in region II.

The multiple phase transitions occur by varying the density (the inverse of headway). Fig. 3 shows the plots of current against density for $a=1.0$. The current (flow) is obtained by averaging the number of vehicles passing a point over sufficiently large time. The current (flow) is obtained by averaging the number of vehicles passing a point over sufficiently large time. The traffic is classified into five states: (I) free traffic (phase 3), (II) coexisting phase between phases 3 and 2, (III) homogeneous traffic (phase 2) at intermediate density, (IV) coexisting phase between phases 2 and 1, and (V) homogeneous congested traffic (phase 1) at high density. The distinct traffic states $\mathrm{I}-\mathrm{V}$ are shown in Fig. 2. In regions II and IV, the density wave appears and propagates backward. The simulation result deviates from the theoretical current-density curve in regions II and IV because the jams occur.

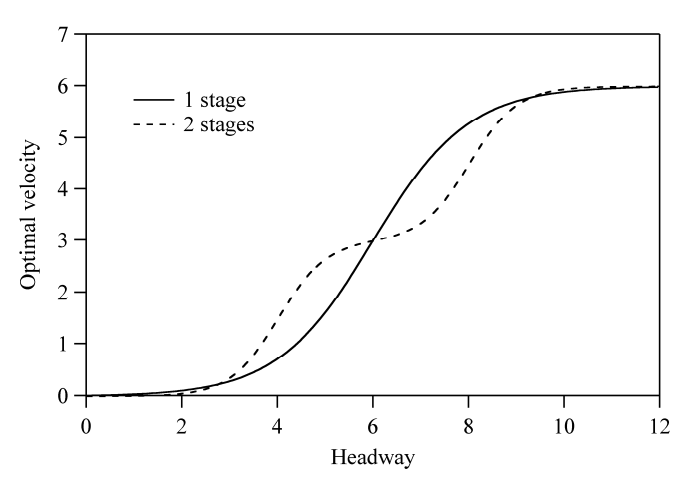

Fig. $1 \mathrm{OV}$ functions for single stage and two stage model.

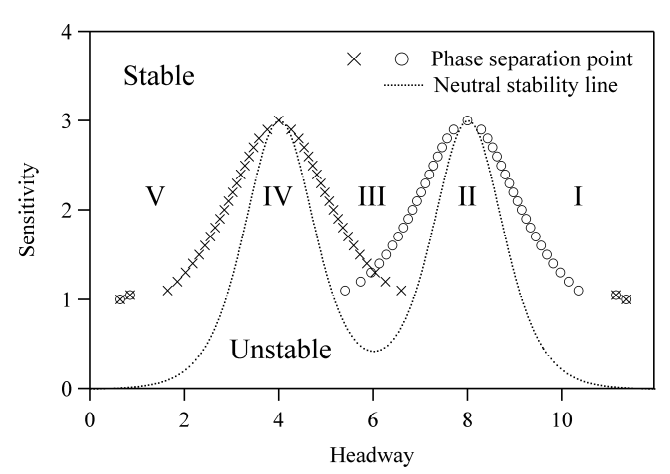

Fig. 2 Phase diagram in the phase space for two stage model.

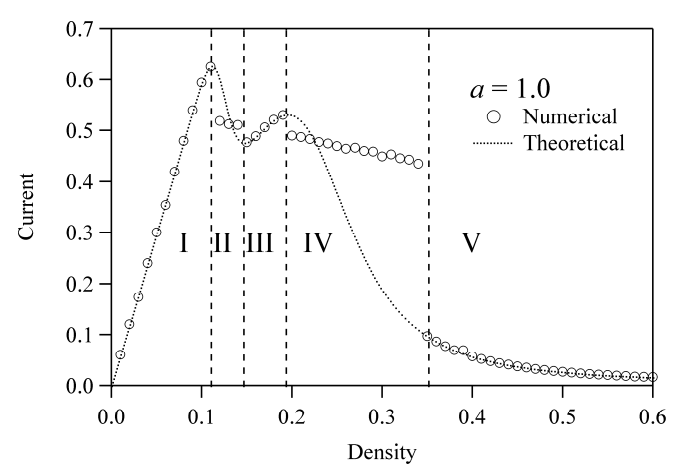

Fig. 3 Plots of current (flow) against density.

\section{References}

[1] T. Nagatani, Rep. Prog. Phys. 65 (2002) 1331.

[2] B. S. Kerner, The Physics of Traffic, Springer, Heidelberg, 2004.

[3] R. Nagai, T. Nagatani, N. Taniguchi, Physica A 350 (2005) 548-562. 